\title{
PROPER MOTION STUDY OF THE GALACTIC ROTATION CURVE
}

\author{
B. FUCHS, S. FRINK, S. RÖSER, R. WIELEN \\ Astronomisches Rechen-Institut Heidelberg \\ Mönchhofstraße 12-14, 69120 Heidelberg, Germany
}

\begin{abstract}
The galactic rotation curve has been determined using the space velocities of $197 \mathrm{OB}$ stars associated with $59 \mathrm{HII}$ regions.
\end{abstract}

\section{Introduction}

The galactic rotation curve has been determined for the inner parts of the galactic disk, interior to the solar annulus, from HI measurements using the tangent point method (Burton and Gordon 1978), whereas the outer rotation curve has been determined using among other objects HII regions embedded in molecular cloud complexes (Brand and Blitz 1993). CO radial velocities are converted to circular velocities assuming that the HII regions move on circular orbits around the galactic center. Obviously proper motions of the objects provide independent information on the rotation curve. Recently the PPM catalogue (Röser and Bastian 1991, Bastian et al. 1993, Röser et al. 1994) has been compiled at the Astronomisches Rechen-Institut which is well suited as a broad data base for proper motions of high accuracy.

\section{Data}

We have based our analysis of the galactic rotation curve on the list of HII regions and their associated molecular cloud complexes by Brand and Blitz (1993). The exciting OB stars of the HII regions in the northern hemisphere are tabulated by Georgelin (1975) and can be identified by cross reference numbers. For the exciting OB stars of HII regions in the southern hemisphere finding charts are given by Brand (1986). Using the cross refe- 
rence numbers, spectral types, or accurate stellar positions we have tried to identify as many stars from the source list in the PPM Star Catalogue as possible. The PPM catalogue has been compiled from a comprehensive set of photographic and meridian circle catalogues and contains astrometric data of about 470000 stars with magnitudes down to $V=11$. We have been able to identify 197 OB stars in the PPM catalogue which are associated with 59 HII regions. Using the positions and proper motions of the stars given in the PPM catalogue and the distances and radial velocities given by Brand and Blitz (1993), respectively, we have calculated the positions and velocities of the stars in galactic coordinates. Fig. 1 shows the projections of the positions of the HII regions onto the galactic plane.

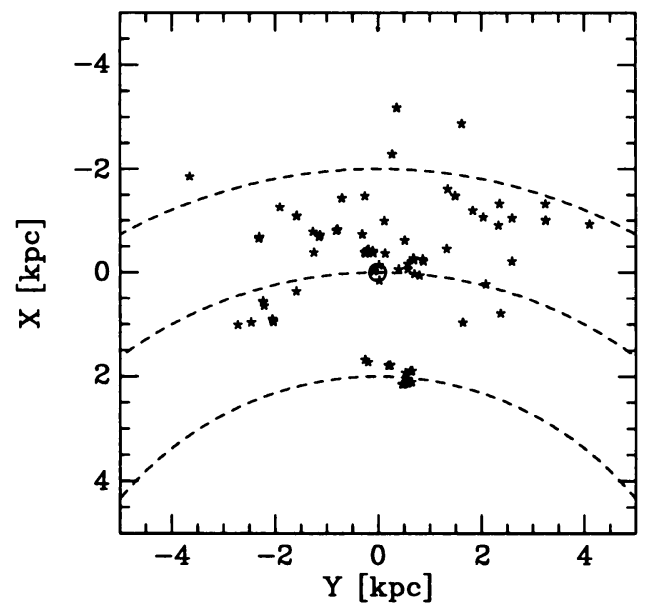

Figure 1. Projection of the positions of the HII regions onto the galactic plane. The dashed lines indicate circles around the galactic center with radii $6.5,8.5$ and $10.5 \mathrm{kpc}$. The position of the sun is indicated as $\odot$.

\section{Results and Discussion}

\subsection{ANGULAR VELOCITY OF THE LSR AND SOLAR MOTION}

The proper motions of the stars are defined in the reference frame of the FK5 (Fricke et al.1988) which represents as closely as possible an inertial frame with its origin at the position of the sun. Since the sun rotates with the local standard of rest (LSR) around the galactic center the proper motions show an apparent rotation of the stars around the LSR with the same angular velocity $\omega_{0}$ as the LSR around the galactic center. Furthermore the solar motion with respect to the LSR has to be taken into account when reducing the velocity data. In order to derive these parameters we have 
fitted a model of the form

$$
\begin{aligned}
& U=-U_{\odot}+\omega_{0} Y-\omega_{0} Y\left(1-R_{\odot} / R\right) \\
& V=-V_{\odot}-\omega_{0} X-\omega_{0}\left(R_{\odot}-X\right)\left(1-R_{\odot} / R\right)
\end{aligned}
$$

to the space velocities of the stars. X, Y are the Cartesian spatial coordinates of the stars relative to the sun. The $\mathrm{X}$-axis points towards the galactic center and the Y-axis into the direction of galactic rotation (cf.Fig.1). U and $\mathrm{V}$ are the corresponding velocities of the stars. The first terms on the right hand sides of equation (1) describe the solar motion with respect to the LSR. The middle terms describe the rigid rotation of the reference frame described above. The rightmost terms model the differential rotation of the Galaxy. Since some of the HII regions are fairly distant from the sun we do not use Oort's approximation but adopt rigorous formulae describing a flat rotation curve. $R$ denotes the galactocentric distance of a star. $R_{\odot}$ is the distance of the sun from the galactic center which we assume as $8.5 \mathrm{kpc}$ throughout the present study. Deviations of the stars from the midplane are ignored. From a $\chi^{2}$-fit of equation (1) to the space velocities of the 197 OB stars we find

$$
\begin{gathered}
\omega_{0}=5.6 \pm 0.4 \mathrm{mas} / \mathrm{a} \\
\left(U_{\odot}, V_{\odot}\right)=(5,6) \mathrm{km} / \mathrm{s} .
\end{gathered}
$$

The value of the angular velocity $\omega_{0}$ which we find corresponds to a circular velocity of $\mathrm{v}_{c}=\omega_{0} \mathrm{R}_{\odot}=224 \mathrm{~km} / \mathrm{s}$ in good agreement with the IAU 1984 value. The estimated error of $\omega_{0}$ has been calculated from the estimated errors given individually for the proper motions of each star in the PPM catalogue and the errors of the radial velocities and the distances of the stars given by Brand and Blitz (1993). Judging from the numerical value of $\chi^{2}$ and the statistical distribution of the velocity residuals we found that the error estimates obtained this way were too low and have increased the error estimates of the proper motions by $30 \%$. This is apparently due to systematic errors of the proper motions in the PPM catalogue which are not fully represented by the individual errors given there. The PPM is tied into the FK5 system. Lindegren (1992) has shown by a systematic comparison of the FK5 system with preliminary HIPPARCOS data that the FK5 positional system has still deficiencies, in particular in the southern hemisphere in a strip at about $\delta=-40^{\circ}$. Unfortunately a number of HII regions at $\ell \approx 260^{\circ}, b \approx 0^{\circ}$ fall into this area, which we have omitted from our analysis. Furthermore it should be kept in mind that in addition to the error sources discussed so far the FK5 system might show further spurious non-physical rotations of the order of 1 mas/a (Schwan 1988).

The solar motion which we find deviates from the classical values of $\left(\mathrm{U}_{\odot}, \mathrm{V}_{\odot}\right)=(9,12) \mathrm{km} / \mathrm{s}$ (Delhaye 1965) which are rejected by our $\chi^{2}$-fit 

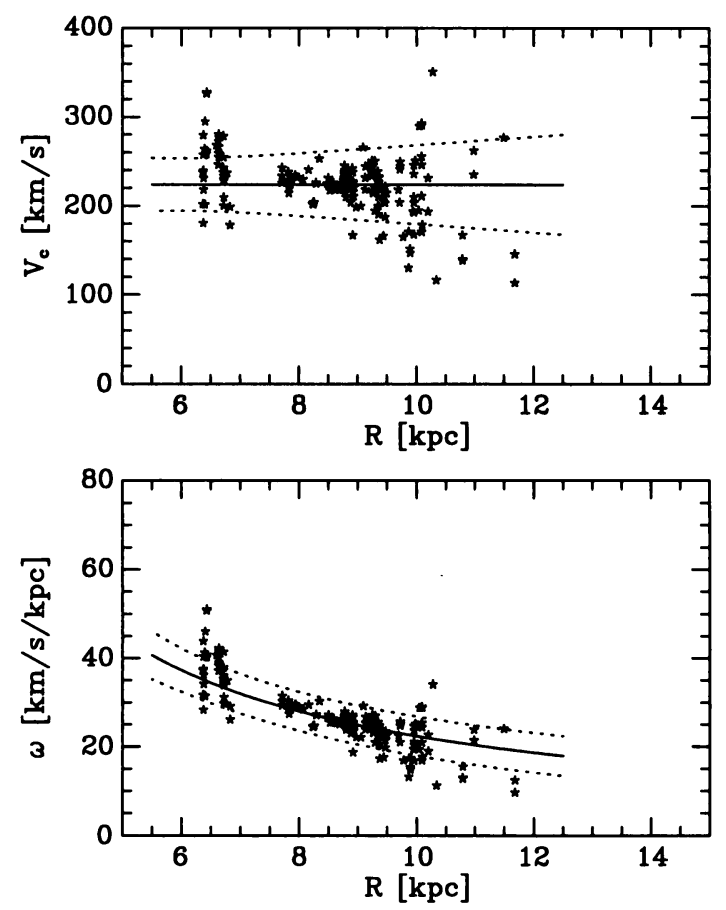

Figure 2. Rotation curve derived from the space motions of $197 \mathrm{OB}$ stars associated with $59 \mathrm{HII}$ regions. The lower panel shows angular velocities as function of galactocentric distance $R$, whereas the upper panel shows circular velocities calculated assuming $R_{\odot}=$ $8.5 \mathrm{kpc}$. The solid lines indicate the rotation law of a flat rotation curve. The dotted lines indicate error estimates based on the errors of the proper motions, radial velocities and distances of the stars.

to the data at a $4 \sigma$ confidence level. The value of $\mathrm{V}_{\odot}$ is closely related to the asymmetric drift of the sample of stars under consideration. Jahreiß and Wielen (1983) have found $\mathrm{V}_{\odot}=5 \mathrm{~km} / \mathrm{s}$ relative to the youngest stars in the solar neighbourhood in close agreement with the value derived here. The value of $\mathrm{U}_{\odot}=5 \mathrm{~km} / \mathrm{s}$ which we use instead of $9 \mathrm{~km} / \mathrm{s}$ corresponds to the apparent inward motion of all $\mathrm{OB}$ stars at about $4 \mathrm{~km} / \mathrm{s}$ relative to the LSR which was noted previously by Fich, Blitz and Stark (1989).This effect is usually interpreted as an outward motion of the LSR (Blitz and Spergel 1991).

\subsection{ROTATION CURVE}

Once the velocities have been corrected for the solar motion and the rotation of the reference frame it is straightforward to determine the circular velocity of each star. Fig. 2 shows the resulting rotation curves derived from the 


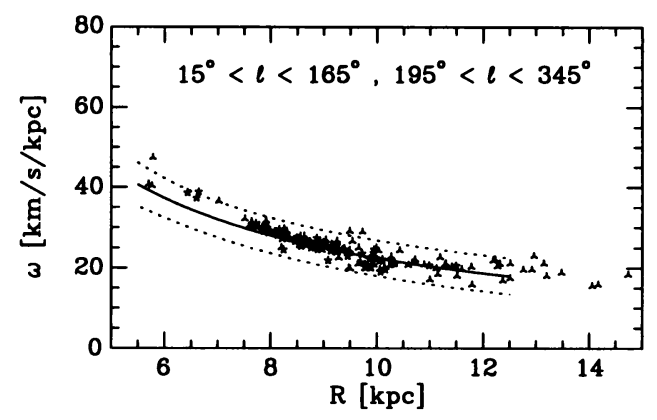

Figure 3. The rotation curve derived solely from the radial velocities of the HII regions assuming circular orbits around the galactic center. The asterisks indicate the 49 HII regions for which we could derive proper motions. The rotation law and error estimates, shown as an aid to the eye, are that of Fig. 2.

space velocities of the 197 OB stars in 59 HII regions. In Fig. 3 we show essentially the rotation curve of Brand and Blitz (1993), derived solely from the radial velocities of the HII regions assuming that they move on circular orbits around the galactic center. HII regions near $\ell=0^{\circ}$ or $\ell=180^{\circ}$ have been excluded. The limitations of proper motion studies become apparent if one realizes that we were only able to derive proper motions for 49 of the 193 HII regions. The rotation curves determined using either the full space velocities or the radial velocity components, respectively, agree very closely and are consistent with a flat shape of the rotation curve or $\omega=\mathrm{v}_{c} / \mathrm{R}$. Finally we have determined Oort's constant using stars with $\left|R-R_{\odot}\right|<1$ $\mathrm{kpc}$ and find $\mathrm{A}=14.4 \pm 1.2 \mathrm{~km} / \mathrm{s} / \mathrm{kpc}$ which agrees nicely with the flat shape of the rotation curve over larger distances intervals. The second Oort constant is according to this determination $B=-12.1 \pm 1.2 \mathrm{~km} / \mathrm{s} / \mathrm{kpc}$.

\subsection{RESIDUAL VELOCITIES}

The orientations of the velocity residuals after subtraction of the systematic velocity components due to the flat rotation curve from the space velocities of the stars are shown in Fig. 4 projected onto the galactic plane. Most of the velocity residuals are randomly orientated with the exception of the Perseus spiral arm. There is a trend of coherent motion along the spiral arm with the effect that the stars in the spiral arm tend to lag behind the general rotation of the disk. Exactly such a behaviour is predicted by the density wave theory of spiral structure for stars born recently in the shock front of the interstellar gas included by the spiral density wave (Shu et al. 1972).

Finally we have looked in our data for an apparent tilting of the galactic 
plane, but found in contrast to Miyamoto et al. (1993) no significant effect.

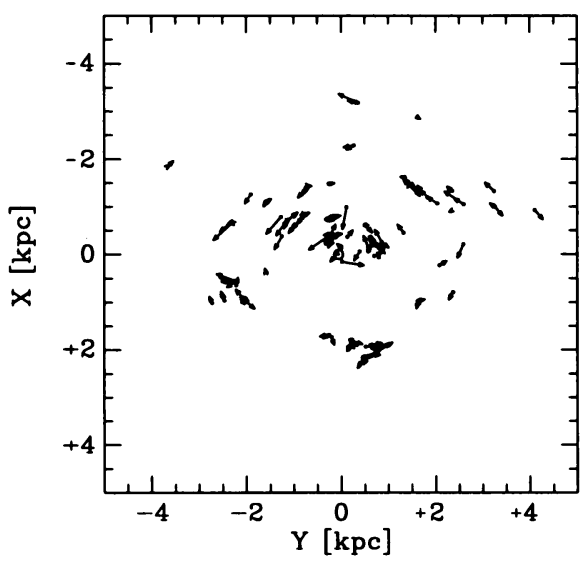

Figure 4. Velocity residuals projected onto the galactic plane after subtraction of a flat rotation curve, normalized by the errrors of the space velocities. One unit of the normalized residuals corresponds to a vector with a length of $150 \mathrm{pc}$ in the diagram. Notice the aligned velocity pattern in the Perseus spiral arm in the upper right hand quadrant of the figure.

\section{References}

Bastian,U., Röser,S., Yagudin,L.I., Nesterov,V.V., Polozhentsev,D.D., Potter,Kh.I., Wielen,R., Yatskiv,Ya.S.: 1993, PPM Star Catalogue Vols.III, IV, Spektrum Akademischer Verlag, Heidelberg

Blitz,L., Spergel,D.N.: 1991, ApJ 370, 205

Brand,J.: 1986, Ph.D. thesis, Univ. of Leiden

Brand,J., Blitz,L.: 1993, AA 275, 67

Burton,W.B., Gordon,M.A.: 1978, AA 63, 7

Delhaye,J.: 1965, in "Galactic Structure", (eds.) A. Blaauw, M. Schmidt, Univ. Chicago Press, p. 61

Fich,M., Blitz,L., Stark,A.A.: 1989, ApJ 342, 272

Fricke,W., Schwan,H., Lederle,T., Bastian,U., Bien,R., Burkhardt,G., du Mont,B., Hering,R., Jährling,R., Jahreiß,H., Röser,S., Schwerdtfeger,H.M., Walter,H.G.: 1988, Veröff. Astron. Rechen-Inst. Heidelberg No. 32

Georgelin,Y.M.: 1975, Ph.D. thesis, Univ. de Provence

Jahreiß,H., Wielen,R.: 1983, IAU Coll. No. 76, 277

Lindegren,L.: 1992, in "Space Sciences with particular emphasis on High Energy Astrophysics", (eds.) T.D. Guyenne, J.J. Hunt, ESA, p. 3

Miyamoto,M., Sôma,M., Yoshizawa,M.: 1993, AJ 105, 2138

Röser,S., Bastian,U.: 1991, PPM Star Catalogue Vols. I, II, Spektrum Akademischer Verlag, Heidelberg

Röser,S., Bastian,U., Kuzmin,A.: 1994, AA Suppl. 105, 301

Schwan,H.: 1988, AA 198, 116

Shu,F.H., Milione,V., Gebel,W., Yuan,C., Goldsmith,D.W., Roberts,W.W.: 1972, ApJ 173,557 


\section{DISCUSSION}

A. Gould: The results you obtain by $\mathrm{W}_{o}$ is substantially at odds with that found by measuring the proper motion of $\mathrm{Sgr} \mathrm{A}^{*}$. Have you considered what distortion of the galaxy might account for this?

Fuchs: -

H. van Woerden: The difference between the angular speed derived by Fuchs and that by Backer and Sramek from $\mathrm{SgrA}^{*}$ is only $1.25 \pm 0.52 \mathrm{mas} / \mathrm{yr}$, less than $3 \sigma$ Taken at face value, it could be explained by a motion of $\operatorname{Sgr}{ }^{*}$ relative to the dynamic center of the Galaxy at $40 \mathrm{~km} / \mathrm{sec}$.

Fuchs: A valid point. One might add that the reference frame of the PPM Fk5 might still show non-physical rotations of the order of 1 mas/yr which will be removed once the extragalactic link is established. 